\title{
Trace Vapor Testbed and Vapor Generators for Hazardous Chemicals, Explosives and Narcotics
}

\author{
Susan L. Rose-Pehrsson ${ }^{1}$, Greg E. Collins ${ }^{1}$, Braden C. Giordano ${ }^{1}$, Mark H. Hammond ${ }^{1}$, Cy R. \\ Tamanaha1, Michael P. Malito ${ }^{2}$, and Christopher Katilie' ${ }^{2}$, \\ ${ }^{1}$ Naval Research Laboratory, Chemistry Division, 4555 Overlook Ave, Washington, DC. USA, \\ 2 Nova Research, Inc. 1900 Elkin St., Suite \#230, Alexandria, VA 22308, USA \\ rosepehrsson@nrl.navy.mil
}

\begin{abstract}
:
Trace vapor detection of hazardous chemical vapors, explosives and narcotics is critical to the protection of warfighters and travelers from explosive devices, and control of borders to prevent illegal smuggling of narcotics or cargo. Sensor and instrument developers are developing new materials and sensors for hazardous chemicals, explosives and narcotics vapor detection. However, uniform, reliable methods for evaluating new detection systems are limited, making assessments of the new products difficult. Many explosives and narcotics have extremely low vapor pressures, which makes trace vapor generation and transport an extremely difficult task.

The US Naval Research Laboratory has designed, and constructed a state-of-the-art Trace Explosives Sensor Testbed (TESTbed) system to evaluate new materials, sensors and instrumentation for hazardous vapor detection. It quantitatively delivers trace vapors to as many as six sensors or materials, simultaneously. The TESTbed includes a zero-grade air source with temperature and relative humidity control, and vapor generation systems for a wide variety of chemicals and potential interferents. The TESTbed has dedicated computer control of a standardized vapor delivery system with an automated data collection system suitable for obtaining high quality validation data. In addition, a compact version of the TESTbed, the Trace Vapor Generator for Explosives and Narcotics (TV-Gen) has recently been developed. The TV-Gen is capable of reproducibly and accurately generating trace vapors (parts per quadrillion to parts per million) of low vapor pressure compounds such as explosives and narcotics for delivery to sensors and materials undergoing testing or calibration.
\end{abstract}

Key words: hazardous vapor generation, explosives, narcotics, sensor testing, vapor concentration validation.

\section{Trace Explosives Sensor Testbed (TESTbed)}

A novel vapor delivery testbed, referred to as the Trace Explosives Sensor Testbed, or TESTbed, was developed that is amenable to both high- and low-volatility hazardous vapors including explosives, narcotics and toxic industrial chemicals, see Figure 1. The TESTbed incorporates a six-port dual-line manifold system housed in an oven that can be operated up to $130{ }^{\circ} \mathrm{C}$. The dual manifold can be rapidly switched between a dedicated clean air source and a trace analyte vapor source as shown in Figure 2. Vapors can be sourced through a number of means including gas cylinders, permeation tube ovens, dynamic headspace chambers, and a Pneumatically Modulated Liquid Delivery System (PMLDS) coupled to a perfluoroalkoxy total-consumption microflow nebulizer. Key features of the
TESTbed include continuous and pulseless control of trace vapor concentrations with wide dynamic range of concentration generation, six sampling ports with reproducible vapor profile outputs, limited low-volatility explosives adsorption to the manifold surface, temperature and humidity control of the vapor stream, and a graphical user interface for system operation and testing protocol implementation. The TESTbed has been demonstrated for explosives, narcotics and toxic industrial chemicals, including extenisve studies of the following explosives: nitromethane (NM), nitroglycerine (NG), ethylene glycol dinitrate (EGDN), triacetone triperoxide (TATP), 2,4,6trinitrotoluene (TNT), pentaerythritol tetranitrate (PETN), and hexahydro-1,3,5-trinitro-1,3,5triazine (RDX).[1] An on-line validation system attached to a sample port provides near real- 
time verification of the explosives from part-permillion (ppm) to part per quadillion (ppq) levels, see Figure 3.

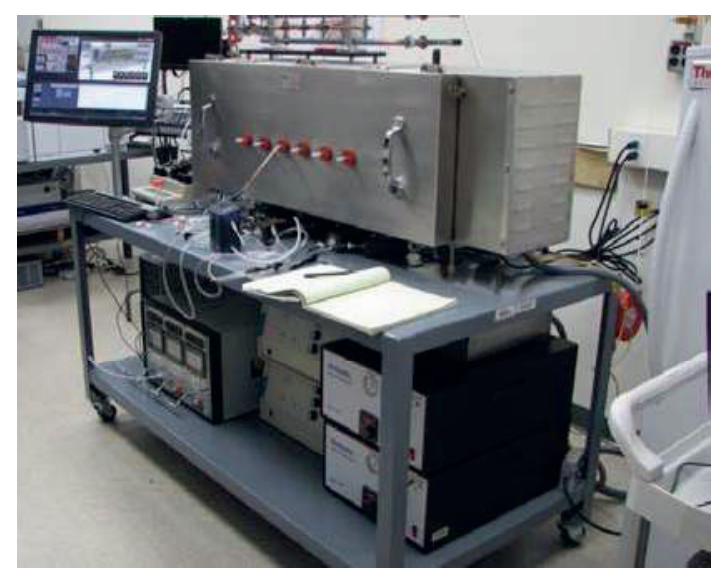

Fig. 1. The TESTbed has dedicated computer control of a standardized vapor delivery system with an automated data collection system suitable for obtaining high quality data for sensor validation.

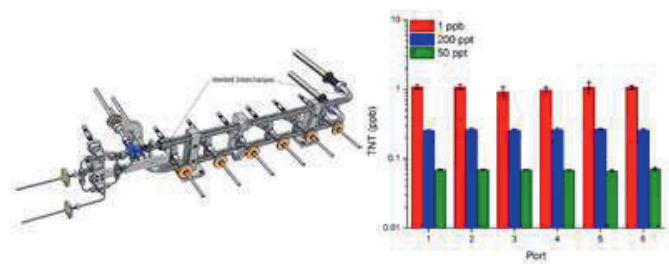

Fig. 2. The TESTbed has a Sulfinert ${ }^{\mathrm{TM}}$ treated stainless steel dual distribution manifolds that provides rapid switching from clean to analyte vapor stream. It has two vapor inputs and 6 sample ports. The manifold is easily removable and exchanged to eliminate cross contamination issues. The 6 sample ports provide reproducible vapor profile outputs as shown here for TNT.

\begin{tabular}{|c|c|c|c|c|c|}
\hline Explosive & Sat. Vapor Conc.* & \multicolumn{2}{|c|}{ TDS-CIS-GC } & \multicolumn{2}{c|}{ On-line CIS-GC } \\
\hline & & Sample Time (Vol.) & LCQ & Sample Time (Vol.) & LCQ \\
\hline TNT & $\sim 9 \mathrm{ppb}_{\mathrm{v}}$ & $60 \mathrm{~min}(6 \mathrm{~L})^{\mathrm{a}}$ & $3.4 \mathrm{ppt}$ & $4 \mathrm{~min}(0.66 \mathrm{~L})^{\mathrm{b}}$ & $100 \mathrm{ppq}$ \\
\hline $\mathrm{RDX}$ & $\sim 5 \mathrm{ppt}_{\mathrm{v}}$ & $60 \mathrm{~min}(6 \mathrm{~L})^{\mathrm{a}}$ & $4.3 \mathrm{ppt}$ & $4 \mathrm{~min}\left(0.66 \mathrm{~L}^{\mathrm{b}}\right.$ & $500 \mathrm{ppq}$ \\
\hline PETN & $\sim 11 \mathrm{ppt}_{\mathrm{v}}$ & $30 \mathrm{~min}\left(3 \mathrm{~L}^{\mathrm{a}}\right.$ & $12 \mathrm{ppt}$ & & \\
\hline TATP & $\sim 63 \mathrm{ppm}_{\mathrm{v}}$ & & & $1 \mathrm{~min}(0.025 \mathrm{~L})^{\mathrm{a}}$ & $5.5 \mathrm{ppb}$ \\
\hline $\mathrm{NM}$ & $\sim 46,800 \mathrm{ppm}_{\mathrm{v}}$ & & & $0.5 \min (0.0125 \mathrm{~L})^{\mathrm{a}}$ & $3000 \mathrm{ppb}$ \\
\hline EGDN & $\sim 102 \mathrm{ppm}_{\mathrm{v}}$ & & & $1 \min (0.1 \mathrm{~L})^{\mathrm{a}}$ & $3.0 \mathrm{ppb}$ \\
\hline NG & $\sim 645 \mathrm{ppb}_{\mathrm{v}}$ & & & $1 \min (0.1 \mathrm{~L})^{\mathrm{a}}$ & $3.0 \mathrm{ppb}$ \\
\hline
\end{tabular}

Fig. 3. A summary of the explosive vapor concentrations validated by collecton on Tenax tubes and analyzed by gas chromatography (GC) and using an online cryotraped GC/MS. Lower vapor concentrations are achievable with longer sample times.

\section{Trace Vapor Generator for Explosives and Narcotics (TV-Gen)}

Building on the lessons learned with the TESTbed, a portable, compact vapor generation system, the Trace Vapor Generator for Explosives and Narcotics (TV-Gen) has been developed that reproducibly and accurately generates trace vapors of low vapor pressure compounds such as explosives and narcotics in the parts per quadrillion to parts per million range. The TV-Gen consists of an oven that can be heated up to $130{ }^{\circ} \mathrm{C}$ and a control box as shown in Figure 4 . It provides a single output from a dual manifold (Figure 5 left) that can be easily switched between clean air and trace levels of analytes. Trace explosives and narcotics are introduced into a diluent air vapor stream via nebulization, which is an effective way of vaporizing compounds that otherwise decompose at elevated temperatures. The humidity and analyte introduction are achieved using the custom PMLDS system (Figure 5 right) developed for the TESTbed. The manifold is contained within the oven to ensure minimal adsorption of analytes/explosives to the surfaces. The manifold system was designed to be easily exchanged and can be totally disassembled for thorough cleaning.

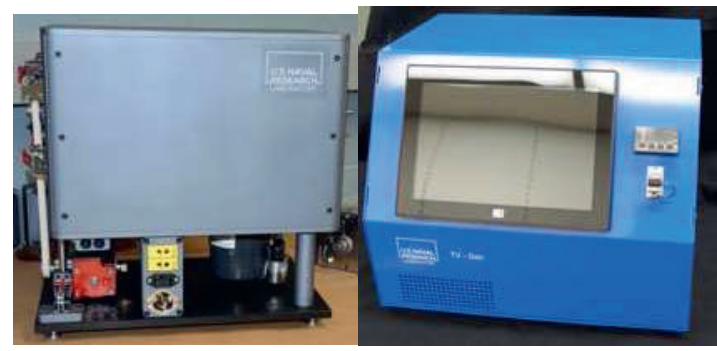

Fig. 4. The TV-Gen oven and the control box

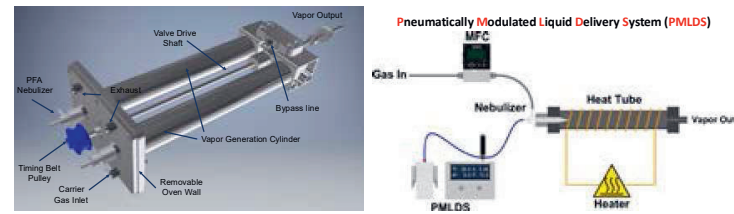

Fig. 5. The dual manifold and PMLDS system.

The system has been fully characterized using the explosives shown in Figure 3. The TV-Gen was recently used to test an explosive detection system based on ion mobility spectrometry.

\section{References}

[1] G.E. Collins, M.P. Malito, C.R. Tamanaha, M.H Hammond, B.C. Giordano, A.L. Lubrano, C.R. Field, D.A. Rogers, R.A. Jeffries, R.J. Colton, and S.L. Rose-Pehrsson, "Trace explosives sensor testbed (TESTbed,)" Review of Scientific Instruments 88, 034104 (2017); doi: $10.1063 / 1.4978963$

This work was supported by the US Department of Homeland Security, Science and Technology Directorate, Homeland Security Advanced Research Projects Agency, Explosives Division 